\title{
ELECTROMOTIVE DRUG ADMINISTRATION OF LIDOCAINE AS AN ALTERNATIVE ANESTHESIA FOR TRANSURETHRAL SURGERY
}

\author{
MICHAEL A. S. JEWETT,$*$, LUC VALIQUETTE, $\dagger$ HEATHER A. SAMPSON, $\dagger$ JOEL KATZ,$\dagger$ \\ YVES FRADET AND DONALD A. REDELMEIER
}

From the Divisions of Urology, Departments of Surgery, Universities of Toronto, Montreal and Laval, Montreal and Laval, Quebec, Departments of Psychology and Medicine, University of Toronto, Division of Clinical Epidemiology, Sunnybrook Health Science Centre and Toronto Hospital, Toronto, Ontario, Canada

\section{ABSTRACT}

Purpose: A multicenter study was undertaken to evaluate the safety, efficacy and cost of electromotive drug administration of intravesical lidocaine to produce bladder local anesthesia as an alternative to traditional methods of spinal or general anesthesia.

Materials and Methods: A total of 94 patients were enrolled in the study who had either a history of bladder tumor that required cold cup bladder biopsy with fulguration for possible recurrence as a comparison trial, a bladder tumor treated with transurethral resection/fulguration or benign prostatic hyperplasia/carcinoma treated with transurethral resection. Pain scores using a Verbal Rating Scale were recorded for each individual biopsy, fulguration and resection event. Data for direct and indinect costs were collected using a standardized form for each patient to capture the details of the procedure, including times, drugs and disposables for each patient.

Results: There was a significant reduction in pain for patients who received electromotive intravesical lidocaine compared to no anesthesia for biopsy $(p<0.03)$. Similarly, electromotive intravesical lidocaine for bladder biopsy and transurethral bladder tumor resection/fulguration was associated with higher patient satisfaction compared to previous treatments $(p<0.00002)$. In contrast, electromotive intravesical lidocaine was insufficient for 3 of 6 transurethral prostatic resections. The cost per patient was about $\$ 146 \mathrm{Cdn}$ less with electromotive intravesical lidocaine than with conventional general/spinal anesthesia.

Conclusions: Electromotive intravesical lidocaine may be a safe, effective and affordable form of anesthesia for the ambulatory care of patients requiring transurethral bladder biopsy, resection or fulguration with a potential for cost savings.

KEY WORDS: anesthesia, iontophoresis, prostatic neoplasms, bladder neoplasms

Traditional techniques for transurethral resection use general and spinal anesthesia that is typically applied in an inpatient environment. Most of the procedures are of short duration but, given the complexity of these forms of anesthesia, many are undertaken in the operating room and costs are high. Locoregional anesthetic alternatives include intraurethral, ${ }^{1}$ intravesical lidocaine, ${ }^{2,3}$ transurethral needle blocks to urethra, bladder neck and bladder wall, 4 and percutaneous periprostatic and pudendal nerve/saddle blocks. However, attempts to block pain from the bladder with intravesical lidocaine have not been widely adopted because of the short duration of anesthesia and generally poor passive diffusion across the impermeable urothelium. ${ }^{5}$ Electromotive drug administration offers a solution to these limitations.6,7 Significant benefits could be achieved by reducing main operating room time and minimizing the need for inpatient stay. Furthermore, current anesthesia techniques have associated risks and side effects, particularly in an elderly population.

Locoregional anesthesia for transurethral surgery may be possible with electromotive administration of intravesical lidocaine to enhance drug delivery. 6 A direct pulsed current across the bladder mucosa moves high concentrations of ionized lidocaine into the bladder wall by several electrokinetic forces. In particular, drug penetration is enhanced by iontophoresis (active transport of ionized drug down an electrical gradient of appropriate polarity), electro-osmosis (ionized or nonionized drug transport accelerated by convective flow of water due to current induced movement of other ions) and electroporation (increased permeability due to direct electrical effects on biological membranes that permit increased transport down concentration gradients).$^{8-10}$ Iontophoresis is the predominant electrokinetic force involved in the transport of local anesthetic drug. ${ }^{11}$

Electromotive drug administration has been studied in animal systems, and used to deliver intravesical local anesthetic agents for transurethral surgery. Examples include transurethral microwave thermotherapy of benign prostatic hyperplasia, hydrostatic distention of the bladder for interstitial cystitis, delivery of mitomycin and $\delta$-aminolevulinic acid for bladder cancer, and oxybutynin for hyperreflexia. ${ }^{6,12-16}$ Transdermal electromotive application of drugs, including morphine and dexamethasone as well as many other agents, has also been reported. ${ }^{9}$

\section{MATERIALS AND METHODS}

A multicenter study of 94 patients was undertaken to assess the safety, efficacy and cost-effectiveness of electromotive administration of intravesical lidocaine as an alternative to spinal or general anesthesia. Three centers and 3 groups of patients participated in the study. Group 1 comprised 45 patients who required cold cup bladder biopsy with (27) or without (18) electromotive intravesical lidocaine as a comparison trial of electromotively administered lidocaine versus no anesthesia. These 2 consecutive cohorts represented all 
biopsy patients during the study period and all underwent fulguration of the biopsy sites. This group also included 6 patients with concurrent bladder tumor and interstitial cystitis who required bladder biopsy/hydrodistention. Group 2 consisted of 43 patients undergoing transurethral resection/ fulguration who were offered electromotively administered lidocaine as an alternative to general or regional anesthesia. Group 3 included 6 men with benign prostatic hyperplasia (BPH)/carcinoma of prostate undergoing transurethral resection who agreed to be treated with electromotively administered lidocaine.

Patients with known enlargement of the median prostate lobe were excluded from study because of potential difficulty inserting the stiffer catheter containing the electrode. Patients with active hematuria and inflammatory/urinary tract infections also were excluded from study to avoid possible excess lidocaine absorption. In addition, known allergy or sensitivity to any of the medications used, apparent psychological instability and pregnancy were exclusion criteria.

The generator was a battery powered, current controlled, programmable Physionizer $30^{*}$ with a range of 0 to $30 \mathrm{~mA}$. and options of constant or pulsed direct current $(2.5 \mathrm{kHz}$.), the latter being more efficient.6,11 The transvesical transmission of current was achieved with the commercially available CE-DAS* catheter of traditional Foley design but containing a central silver coated electrode acting as an anode. For women the catheter was modified with 3 pairs of opposing side holes in the tip distal to the balloon to allow adequate exposure of the electrode. For men the catheter had a similar series of side holes in the prostatic urethral section plus a pair of larger side holes in the region of the bladder neck.

Patients were instructed to restrict fluids 6 to 12 hours before the procedure to minimize urinary dilution of the lidocaine instillate and introduction of charge competitive ions. As clinically indicated, 1 to $2 \mathrm{mg}$. sublingual lorazepam were administered as an anxiolytic to $50 \%$ of the biopsy and $90 \%$ of the transurethral bladder tumor resection/fulguration patients. Clinical judgment was used to select the percentage of electromotive intravesical lidocaine patients and percentage of controls to receive lorazepam. This agent was not observed to alter patient ability to communicate. Anesthetic gel was instilled into the urethra and no additional analgesia was given unless treatment failed. Sodium chloride-free 100 $\mathrm{ml}$. lidocaine $4 \%$ were mixed with $100 \mathrm{ml}$.) sterile water and $2 \mathrm{ml}$. epinephrine $(1 \mathrm{mg} . / \mathrm{ml}$.) for a final concentration of 1:100,000.

The bladder was catheterized with the appropriate male or female catheter, drained and irrigated with $100 \mathrm{ml}$. sterile water to check for hematuria. Immediately $150 \mathrm{ml}$. of the drug solution to maximize even drug distribution at the optimal ratio of surface area-to-volume were instilled. The dispersive cathode electrodes were placed on abdominal or thigh skin that had been degreased with alcohol wipes and a 2 to 5 $\mathrm{mm}$. layer of conductive gel was applied. Air bubbles were eliminated by pressing and sliding the saline impregnated dispersive electrodes gently side to side to avoid skin injury. The pulsed current was then activated and increased progressively ( 40 to $60 \mu \mathrm{A}$. per second to a maximum of $25 \mathrm{~mA}$. for 20 to 25 minutes, total charge about $600 \mathrm{~mA}$.). Every 5 minutes the catheter was rotated 90 degrees to expose fresh areas of the prostatic urethra (for consistency, this was also done in women). About $50 \mathrm{ml}$. were withdrawn at the first quarter turn and reinfused to circulate the instillate and check for hematuria. About 50 to $60 \mathrm{ml}$. instillate were removed at the second turn to minimize dilution by new urine and replaced with $50 \mathrm{ml}$. fresh drug solution.

Safety assessment was done by calculating an American Society of Anesthesia score and recording adverse events for each patient. A numeric pain score was measured before,

\footnotetext{
* Physion Srl., Mirandola, Italy.
}

during and after the procedures using an 11-point numeric rating scale with end points labeled 0 (no pain) and 10 (worst possible pain).17 Patients were familiarized with the pain scale before treatment and asked to provide a rating between 0 and 10 of the pain they experienced at baseline (before, during and after insertion of the cystoscope), at each biopsy or tumor excision (before, during and after), during coagulation or resection and at discharge from the cystoscopy suite. Patients were also asked to score the overall level of comfort from 0 to 10 and rate whether they would repeat the procedure in the same manner using the same technique of anesthesia.

Demographic features and clinical variables were recorded as mean, standard deviation, median and interquartile range or frequency. For bladder biopsy (group 1) demographic features and clinical variables were compared between the electromotive intravesical lidocaine and control groups by unpaired $t$ test for ratio scale data. Chi-square test with Yates' continuity correction was used to analyze frequency data. To get a comprehensive evaluation of pain, data were summarized as median pain and peak pain with interquartile range. Pain scores were compared between the groups by the Wilcoxon test. The proportion of patients willing to undergo a repeat procedure was compared between the groups by chisquare test with Yates' continuity correction. Two-tailed tests were used with $p<0.05$ considered statistically significant.

Given the nascent character of the technology, preliminary economic estimates were derived from modeling rather than direct observation. Cost data were collected using a standardized form for each patient to capture the details of the procedure, including times, drugs and disposables. The economic analysis was done from the societal perspective and all amounts were expressed in 1997 Canadian dollars. The 5 simplifying assumptions essential for the analysis were 1) iontophoresis saves money by avoiding conventional anesthesia, 2) iontophoresis costs money primarily through the consumption of disposables and partially through the cost of acquiring durable equipment, 3 ) the procedure when effective is as successful as conventional anesthesia and yields similar downstream outcomes, 4) the procedure when not effective allows for switching the patient over to traditional anesthesia with no extra difficulties and 5) the marginal cost of extra time related to using iontophoresis is negligible. The cost of spinal and general anesthesia was estimated by considering the typical charge for an anesthesiologist and the typical expenditures for the operating room, medications in the operating room and post-anesthetic monitoring. The main assumption is that these costs would be avoided by iontophoresis. Anesthesiologist charges were obtained from the Ontario Ministry of Health Schedule of Benefits. Medication costs were obtained from The Toronto Hospital pharmacy. Miscellaneous expenses, such as intravenous sets, electrocardiogram leads and paper records, were assumed to be sufficiently similar in spinal and electromotively delivered anesthesia to be excluded from calculations of marginal costs.

\section{RESULTS}

Most patients reported local tingling sensations when the current was applied and had some cutaneous erythema at the site where the dispersive cathode electrode was attached to the skin. Both reactions disappeared within 1 hour except in 1 patient who experienced a second degree burn at 1 of 2 electrode sites, which subsequently healed spontaneously. Mucosal blanching or "branding" was noted at the bladder neck or in the prostatic urethra in a few patients where the electrode was touching the mucosa at the catheter eyelet. No distortions were noted in the histology of any of the biopsy specimens that could be attributed to iontophoresis or lidocaine. 
Demographic and clinical variables for group 1 were similar for those who did or did not receive electromotive intravesical lidocaine (control group) (table 1). Pain levels were similar before the procedures but were significantly less intense for the electromotive drug administration group than the control group during insertion of the cystoscope, biopsy and coagulation (table 2). Also peak pain during biopsy was significantly less in the electromotive than the control group. A significantly greater proportion of patients in the electromotive than in the control group stated that they would be willing to repeat the procedure (table 1).

For the group 2 patients undergoing transurethral bladder tumor resection/fulguration demographic, clinical variables, and median and peak pain scores during the procedure are shown in tables 3 and 4 . Except for mild pain during cutting (median pain score 1.4), median pain scores for the entire procedure were 0 . However, $25 \%$ of patients had pain scores greater than 3 during tumor excision and $25 \%$ had peak pain scores greater than 5 . The majority of patients $(86 \%)$ stated that they would be willing to repeat the procedure. Of the group 3 patients who underwent transurethral prostatic resection with iontophoresis 3 experienced more discomfort than those undergoing bladder procedures. The remaining 3 patients had remarkably pain-free procedures.

The cost of electromotive intravesical lidocaine included purchasing 1 generator $(\$ 2,600), 1$ specially designed electrode $(\$ 250)$, anesthetic $(\$ 4.70$ per 50 cc vial lidocaine $4 \%$ ) and other medications $(\$ 0.50$ per vial epinephrine $1: 1,000)$. The analysis assumed that the generator can be used for at least 100 treatments before needing replacement or repair and, hence, generator costs per treatment were $\$ 26$ or less. One nonreusable catheter was assumed to be used for each treatment, along with 2 vials of lidocaine and 2 vials of epinephrine, leading to estimated marginal costs of $\$ 286$. The costs for doing the same procedures in the operating room with spinal or general anesthesia were estimated and included $\$ 120$ for an anesthesiologist, $\$ 212$ for the operating room, $\$ 50$ for medications in the operating room and $\$ 50$ for postanesthetic monitoring, for a total of $\$ 432$. Therefore, electromotive intravesical lidocaine was $\$ 146$ cheaper to perform $(\$ 432-\$ 286)$

\section{DISCUSSION}

In this study the 70 patients who underwent bladder biopsy and transurethral bladder tumor resection/fulguration received good anesthesia but 3 of the 6 men who underwent transurethral prostatic resection with electromotive intravesical lidocaine experienced substantial discomfort and the result was unpredictable. Gürpinar et al noted that in a canine model using iontophoresis and methylene blue, dye penetration was less in the bladder neck and prostate compared to the bladder wall.11 The same result might be expected with lidocaine in humans although the catheter is designed to expose this area to the current. Further modifi-

TABLE 1. Demographic features and clinical variables for group 1 patients pretreated with electromotive intravesical lidocaine or biopsied without anesthesia

\begin{tabular}{lcc}
\hline & $\begin{array}{c}\text { Electromotive } \\
\text { Intravesical } \\
\text { Lidocaine }\end{array}$ & Control \\
\hline No. pts. & 27 & 18 \\
\% Men & 52 & 72 \\
Mean age \pm SD & $62 \pm 13.3$ & $68 \pm 13.3$ \\
Mean kg. wt. \pm SD (range) & $\mathbf{7 5} \pm 19.8(36-88)$ & $75 \pm 15.2(32-85)$ \\
Mean No. biopsies \pm SD & $\mathbf{3 . 1} \pm 1.9$ & $3.3 \pm 1.4$ \\
Mean No. coagulation $\pm \mathrm{SD}$ & $5.8 \pm 9.9$ & $6.3 \pm 7.5$ \\
\% Repeat procedure: & & 23 \\
Yes & $\mathbf{8 5 *}$ & 76 \\
No & $\mathbf{1 5}$ & \\
\hline *Chi-8quare p $<0.0002$. & &
\end{tabular}

TABLE 2. Efficacy of electromotive intravesical lidocaine expressed as 0 to 10 rating scores at various times before, during and after bladder biopsy

\begin{tabular}{|c|c|c|c|c|}
\hline & \multicolumn{2}{|c|}{$\begin{array}{c}\text { Electromotive } \\
\text { Intravesical Lidocaine }\end{array}$} & \multicolumn{2}{|c|}{ Control } \\
\hline & $\begin{array}{c}\text { Median } \\
\text { (IQ range) }\end{array}$ & $\begin{array}{c}\text { Peak } \\
\text { (IQ range) }\end{array}$ & $\begin{array}{c}\text { Median } \\
\text { (IQ range) }\end{array}$ & \multirow{2}{*}{$\begin{array}{c}\begin{array}{c}\text { Peak } \\
\text { (IQ range) }\end{array} \\
-\end{array}$} \\
\hline & $(0-0)$ & - & $(0-0)$ & \\
\hline Insertion* & $0 \quad(0-2)$ & - & $2(1-5)$ & - \\
\hline Before biopsy & $0(0-1.3)$ & $0(0-1.5)$ & $0(0-1.5)$ & $(0-2)$ \\
\hline During biopsy $^{+}$ & $0(0-1)$ & $1(0-2)$ & $3(2-5)$ & $(3.3-6)$ \\
\hline After biopsy & $0 \quad(0-2)$ & $(0-3)$ & $0 \quad(0-2)$ & $2(0-3.5)$ \\
\hline Coagulation ${ }^{\ddagger}$ & $0(0-1.4)$ & D $(0-3)$ & $2(0-4.4)$ & $3(1.5-7.3)$ \\
\hline End of procedure & $0 \quad(0-0)$ & - & $0(0-1)$ & - \\
\hline Discharge & $0 \quad(0-0)$ & - & $0 \quad(0-0)$ & - \\
\hline
\end{tabular}

* Electromotive versus control $p<0.01$ (Wilcoxon test).

'Electromotive versus control for median and peak pain scores $p<0.0001$ (Wilcoxon test).

${ }^{\ddagger}$ Electromotive versus control for peak pain scores $p<0.04$ (Wilcoxon test).

TABLE 3. Demographic features and clinical variables for group 2

\begin{tabular}{lc} 
No. pts. & 43 \\
$\%$ Men & 79 \\
Mean age \pm SD & $69 \pm 10.2$ \\
Mean wt. \pm SD (kg.) & $74 \pm 18.3$ \\
\% Repeat procedure & 86 \\
\hline
\end{tabular}

TABLE 4. Efficacy of electromotive intravesical lidocaine expressed as 0 to 10 rating scores at various times, during and after bladder tumor fulguration/resection

\begin{tabular}{|c|c|c|}
\hline & Median Pain (IQ range) & Peak Pain (IQ range) \\
\hline Baseline & $(0-0)$ & - \\
\hline Insertion & $(0-2)$ & - \\
\hline Before cut & $0 \quad(0-3)$ & $(0-0)$ \\
\hline During cut & $1.4 \quad(0-3)$ & $(1-5)$ \\
\hline After cut & $0 \quad(0-0)$ & $0 \quad(0-0)$ \\
\hline Coagulation & $0 \quad(0-1.9)$ & $0(0-4.8)$ \\
\hline End of procedure & $0 \quad(0-0)$ & - \\
\hline Discharge & $(0-0)$ & 0 \\
\hline
\end{tabular}

cation will be necessary for prostate and bladder neck surgery, such as the adjunctive use of prostate block and intravenous sedoanalgesia. Adverse events were either trivial, such as tingling sensations and minor urothelial coagulation by the electrode, or limited to infrequent skin effects of the cathode. Only 1 patient had any morbidity from this skin effect and that might have been prevented with careful application of the skin pad. We did not measure intravenous lidocaine levels but previous reports indicate that little drug is absorbed from the bladder into the systemic circulation 6,12

We did not evaluate intravesical lidocaine without applied electrical current. We and others have inadvertently used electromotive drug administration without current and the patients experienced pain. ${ }^{7}$ Similarly, reversal of polarity during electromotive lidocaine administration lead to poor pain control. We believe that reports of local bladder anesthesia based on the passive diffusion of intravesical lidocaine provide limited support for the use of lidocaine alone and this has never been widely adopted by urologists. ${ }^{1,2}$ This finding is not surprising as bladder mucosa is the least permeable plasma membrane in the human which is important to its normal function.

It is clear from our experience that patient tolerance of pain varies greatly during bladder biopsy or fulguration and that some may be reassured by efforts to reduce discomfort. Therefore, some patients may appear to benefit from pretreatment instillation of local anesthetic but no controlled trial has been conducted to our knowledge. The principal outcome for comparison was the level of pain perceived by the patients, for which we used a simple, efficient and minimally 
intrusive numeric rating pain scale $(0$ to 10$)$. This measure has been used widely in research settings where a quick index of pain is required and to which a numerical statistic can be assigned. It has been shown to be sensitive to interventions which diminish or augment the experience of pain, to take a matter of seconds to administer and to have excellent validity. ${ }^{17}$ It is important to note that no patient scored received any other analgesia or anesthesia.

The evaluation of new surgical technology requires an assessment of cost-effectiveness and needs to consider what goes on outside the operating room. ${ }^{18}$ Expenditures related to preoperative assessment, followup evaluations and untoward events can be substantial. When estimating the marginal costs of this procedure relative to spinal and general anes. thesia, however, these costs were assumed to be sufficiently stable to contribute equally in both and to disappear in a comparison between the 2 approaches. This assumption is reasonable from a health care perspective but it is somewhat conservative from the individual patient perspective given that the electromotive technique requires little recovery time and faster return to usual activity. Actual costs with general spinal anesthesia will depend on local practice to some extent and may be generally lower in Canada than in the United States.

Our experience suggests that electromotive drug administration will probably not be successful for every patient and that clinicians may initially have problems in identifying who should be included. There will be a learning curve for urologists beginning to use iontophoresis. Failures with iontophoresis are noteworthy, given that spinal and general anesthesia is almost always dependable. In our economic analysis we assumed that when the technique was unsuccessful, patients were switched over to spinal or general anesthesia with no additional difficulty. However, such failures may be relatively expensive, given that they invoke the full cost of both forms of anesthesia. A final limitation of the technique is the slow onset of action. Instillation requires 25 to 30 minutes to allow medication to penetrate the lining of the urinary tract. Assessing the cost of such waiting is problematic. We currently perform the procedures at an outpatient cystoscopy unit, and are persuaded that this anesthetic technique offers advantages in terms of safety and cost while providing near equal levels of anesthesia.

\section{REFERENCES}

1. Dawkins, G. P. C., Harrison, N. W. and Ansell, W.: Urethral anaesthesia with topical bupivacaine. A role for a longeracting agent. Brit. J. Urol., 76: 484, 1995.

2. Thrasher, J. B., Kreder, K. J., Peterson, N. E. and Donatucci,
C. F.: Lidocaine as topical aneathesin for bladder mappings and cold-cup biopsieat. J. Urol., 160: 336, 1993.

3. Pode, D., Zylber-Katz, $E$. and Shapiro, A.: Intravesical lidocaine: topical anesthesia for bladder mucosal biopsies. J. Urol., 148: 95, 1992.

4. Orandi, A.: Urological endowcopic surgery under local anesthe. sia: a cost-reducing idea. J. Urol., 132: 1146, 1984

5. Teruo, M., Watanabe. H. and Kobayashi, T.: Absorption of anticancer drugs through bladder epithelium. Urology, 27: 148. 1986.

6. Fontanella, U. A., Rossi, C. A and Stephen, R. L.: Bladder and urechral anaesthesia with electromotive drug administration (EMDA): a technique for invasive endoscopic procedures. Brit. J. Urol., 78: 414, 1997.

7. Lugnani, F., Mazza, G., Cerulli, N., Rossi, C. and Stephen, R. L.: Iontophoresis of drugs in the bladder wall: equipment and preliminary studies. Artif. Organs, 17: 8, 1993.

8. Gangarosa, L. P. S. and Hill, J. M.: Modern iontophoresis for local drug delivery. Int. J. Pharm., I29; 159, 1995.

9. Stephen, R. L., Miotti, D., Bettaglio, R., Rossi, C. and Bonezzi. C.: Electromotive administration of a new morphine formulation: morphine citrate. Artif. Organs, 16: 461, 1994

10. Prausnitz, M. R., Bose, V. G., Langer, R., and Weaver, J. C.: Electroporation of mammalian skin: a mechanism to enhance transdermal drug delivery. Proc. Natl. Acad. Sci., USA, 80: $10504,1993$.

11. Gürpinar, T., Truong, L. D., Wong, H. Y. and Griffith. D. P.: Electromotive drug administration to the urinary bladder: an animal model and preliminary results. J. Urol., 156: 1496, 1996.

12. Gürpinar, T., Wong, H. Y. and Griffith, D. P.: Electromotive administration of intravesical lidocaine in patients with inter. stitial cystitis. J. Endourol., 10: 443, 1997.

13. DiStasi, S. M., Vespasiani, G., Giannantoni, A., Massoud, R., Dolci, $S$. and Micalli, F.: Electromotive delivery of mitomycin $C$ into human bladder wall. Cancer Rex., 57: 875, 1997.

14. Riedl, C. R., Knoll, M., Plas, E. and Pluger, H.: Intravesical electromotive drug administration: technique, indications and adverse effects. J. Urol., 169: 1851, 1998.

15. Eder, I., Stenzl, A., Kostron, H., Bartsch, G. and Klocker, H.: Electromotive diffusion (EMD) and photadynamic therapy with 3-aminolaevulinic acid (Al.A) for superficial bladder can. cer. J. Urol., part 2, 234A, abstract 21, 1995.

16. DiStasi, S. M., Giannantoni, A., Massoud, R., Cortese, C.. Vesfrasiani, G. and Micali, F.: Electromotive administration of oxybutynin into the human bladder wall. J. Urol., 158: 228, 1997.

17. Jensen, M. P. and Karoly, P.: Self-report scales and procedures for assessing pain in adults. In: Handbook of Pain Assessment. Edited by D. C. Turk and R. Melzack. New York: Guilford Press, p. 135, 1992.

18. Detaky, A. S. and Naglie, I. G.: A clinician's guide to conteffectiveness analyris. Ann. Int. Med., 113: 147, 1990. 